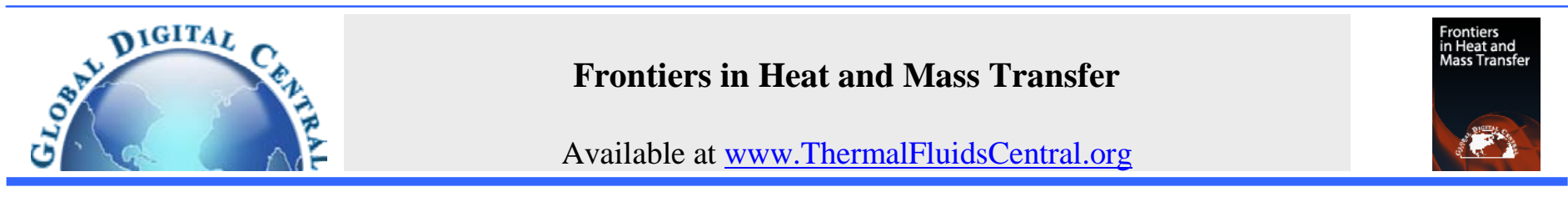

\title{
USING INCOMPLETE VARIABLE CROSS-SECTION HIGHLY CONDUCTIVE INSERTS FOR COOLING A DISC
}

\author{
F. Sharifi ${ }^{\mathrm{a}}$, H. Ghaedamini ${ }^{\mathrm{b},{ }^{*}}$ M.R. Salimpour ${ }^{\mathrm{a}}$ \\ ${ }^{a}$ Department of Mechanical Engineering, Isfahan University of Technology, Isfahan 84156-83111, Iran \\ ${ }^{b}$ Department of Mechanical Engineering, National University of Singapore, 9 Engineering Drive 1, Singapore 117576, Singapore
}

\begin{abstract}
In the present study, conductive cooling of a disc is done by means of incomplete constant and variable cross-section highly conductive inserts embedded in radial and tributary configurations. Variational calculus is invoked to determine the optimum shape of the cross-sections of the inserts. Firstly, it is tried to derive an equation for thermal resistance of the disc for radial configuration of inserts based on the procedure used in constructal studies. This is done by implementing the optimized thermal resistances of elemental sectors. Then, the computed elemental sectors are put together so that they make branching configuration of inserts in the disc. Out of the comparison between the obtained thermal resistances of the disc with constant and variable cross-sections, it is concluded that using variable cross-sections reduces thermal resistance, but this effect differs in radial and tributary configurations, i.e., increasing the complexity of tributary patterns does not always reduce the thermal resistance more effectively in comparison with radial configurations.
\end{abstract}

Keywords: Constructal, variable cross-section, variational, incomplete insert, disc, cooling.

\section{INTRODUCTION}

In today's high-performance microelectronics, one can say for sure that heat dissipation is among the important factors that need special attention as in some cases it acts as a bottleneck which dictates the performance of the system. Moreover, as the length scale of these devices continues to diminish, convective cooling of the component may not be viable due to technical issues. Therefore, conduction of the heat by means of highly conductive inserts emerges as a tool to transfer the generated heat to the edge or the center of the device where attached heat sink can be used to remove this heat. This method is used in this paper for cooling a disc shape body based on constructal theory.

Constructal theory attempts to explain the evolvement of finitesize flow systems through time and it says this evolvement is based on a simple law: for a finite size flow system to survive in time, it should evolve in such a way that it provides easier access to the flow that goes through it. Knowing this law, one not only can explain natural systems but also he can implement it into man-made devices as an attempt to reach to the designs that are more natural, keeping in mind that nature has the answer to lots of our problems. Here, it should be noted that even the natural systems are not duly perfect. Hence, the future does not belong to the perfect, or the ideal, but it belongs to the imperfect designs that are the least imperfect possible (Bejan and Lorente, 2008). This has been the time arrow of design evolution in technology, biology, geomorphology, and social organization in the past years (Bejan, 2000, 2008, 2010; Bejan et al., 2000; Bejan and Lorente, 2006; Bejan and Merkx, 2007).

Pure conductive cooling of electronic components by using highly conductive inserts implemented in low conductive domain is one applicable area of constructal theory which many aspects of it are investigated until now. Ledezma et al. (Ledezma et al., 1997) constructed a cooling network for a rectangular domain with constant volumetric heat generation. They concluded that a tree-shaped network of highly conductive material should be an optimum design. Dan and Bejan (Dan and Bejan, 1998) looked into the problem from a different angle and by changing the geometry of flow path, they tried to minimize the time needed to discharge a volume to a sink by means of a constructal tree network. Almogbel and Bejan (Almogbel and Bejan, 2001) used non-uniform distribution of highly conductive material. By this configuration, they could achieve a significant improvement in global performance. Mathieu-Potvin and Gosselin (Mathieu-Potvin and Gosselin, 2007) proposed an evolutionary algorithm to optimize the thermal resistance and by comparing their results to those predicted previously by constructal theory, they showed several similarities in term of performances and geometries. Gosselin and Bejan (Gosselin and Bejan, 2004) studied tree configuration of highly conductive materials at micro and nano scales and stated that at such a small scale, thermal conductivities vary with the shape and dimension of the system. All of the above works were done for a rectangular domain; however, there are also some investigations for circular (Rocha et al., 2002, 2006) and triangular (Ghodoossi and Egrican, 2004) domains. An analytical solution for radial and bifurcation configuration of highly conductive inserts embedded in a disc was presented by Rocha et al. (Rocha et al., 2002). They believed that highly conductive material inserts can change the direction of heat flux into two perpendicular directions. A later work by Rocha et al. (Rocha et al., 2006) was devoted to a different construction by adding loops to the configuration.

There is a series of studies in which the widths of inserts are optimized using variational calculus. Ledezma et al. (Ledezma et al., 1997) used cross-sectional optimized inserts to minimize resistance of the tree network. Then Rocha et al. (Rocha et al., 2002) utilized this method to optimize the width of radial inserts distributed in a disc domain and further compared thermal resistance and other geometric parameters related to this case to a constant- $D$ assumption. 
Optimizing of variable cross-section highly conductive insert was developed in refs. (Wei et al., 2009; Wei et al., 2010; Zhou et al., 2007) for a rectangular domain. In these investigations based on constructal theory, the thermal resistance was optimized through assembling the constructs in several times using the results of the simpler elemental constructs. Comparison of the results was done in each step by comparing them with simpler architectures with fewer assemblies (Zhou et al., 2007) and also with constant cross-section configurations (Wei et al., 2009). Mentioned results revealed that increasing the complexity of the configuration cannot always decrease the thermal resistance. However it is mentioned that the optimized minimum thermal resistance of variable cross-section cases is smaller than that of constant cross-section. The recent study performed by using constructal entransy dissipation rate minimization method based on discrete variable cross-section highly conductive insert (Wei et al., 2010) showed that as the assembly's order increases, i.e. complexity of the architecture, the minimum mean temperature difference on elemental area with variable crosssection highly conductive insert increases whereas for the case of constant cross-section highly conductive insert, this parameter decreases and for both of them, it approaches a constant as the assembly's order increases.

In the present study, the work on incomplete inserts extending outward from center to a specific distance in a disc (Fig. 1) is further developed by giving another degree of freedom to the geometry; that is, the cross-section change of inserts. By using variational calculus, the incomplete variable cross-section highly conductive inserts in radial and branching configurations used for cooling a disc-shaped body are investigated. Hence, the optimized thermal resistance is determined and compared to the constant cross-section highly conductive insert case. The results showed that for the case under study here, decrease of thermal resistance can be achieved from the case with constant cross section inserts. Moreover, under some circumstances which will be illustrated, the thermal resistance of the disc with incomplete inserts surpasses the complete insert structure. Also by increasing the complexity of the network from radial to branching configurations, there is less decrease in optimized thermal resistance. That means increasing the complexity of the system will diminish the effect of varying cross-section on thermal resistance. However, it should be noted that this is an achievement itself as the price of highly conductive material is a determining factor.

\section{DESCRIPTION OF THE PROBLEM}

Designing a disc-shaped electronic component is considered in this study, Fig. 1. In an analogy to constructal studies, we should define the constraints as well as degrees of freedom; these two will compete against each other to shape the geometry. Usually, global constraint is the volume occupied by the device and in this way, installing as much circuitry as possible in this volume should be the objective of the design. Since from the thermal design point of view, the pure effect of these components is the heat generation, the objective simply means as much heat generation as possible in this specified volume. The local constraint is to prevent the highest temperature of the package, $T_{\max }$, exceeding a specified value. It is obvious that if $T_{\max }$ goes beyond this allowable amount, the function of the local component is threatened. Without any major effect on the generality of the problem and just for the sake of simplicity, it is considered that there is a uniform distribution of heat generation throughout the adiabatic wall bounded disc. To gather heat, highly conductive inserts with the thickness of $D$ are implemented which their material's conductivity, $k_{p}$, is considerably higher than the disc's material with conductivity of $k_{0}$ such that $\tilde{k}=k_{p} / k_{0} \gg 1$. This network of highly conductive inserts then derives the heat to the center of the disc where a heat sink exists to gather that heat. Figure 1 shows a radial configuration of the inserts. The composition of the two-material composite is fixed which is accounted for by defining volume fraction $\phi$ as the volume of $k_{p}$ material to total volume.

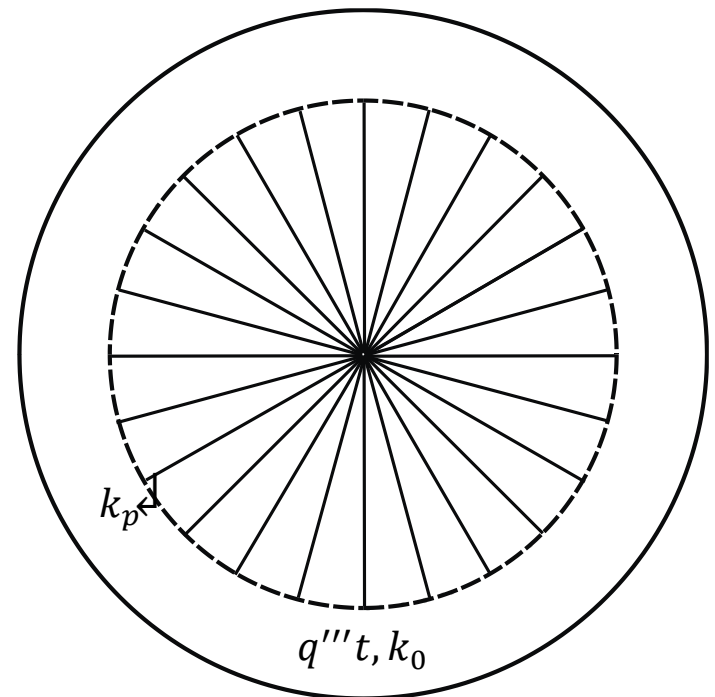

Fig. 1 Incomplete radial configuration of highly conductive inserts embedded in a uniform heat generating disc.

\section{MATHEMATIC FORMULATION}

In this part, using variational calculus, cross-section of the highly conductive insert for radial configuration is optimized and then considering the calculated cross-section shape, it is tried to optimize the thermal resistance. Similarly, the same procedure is implemented for the fractal network. Also, the relation of constant cross-section highly conductive insert is compared with that of variable crosssection highly conductive insert.

\subsection{Radial pattern}

A radial configuration is maybe the simplest structure to design a conductive network to cool a disc. The disc with radial inserts, Fig. 1, is composed of $N$ sectors which are shown in Fig. 2.

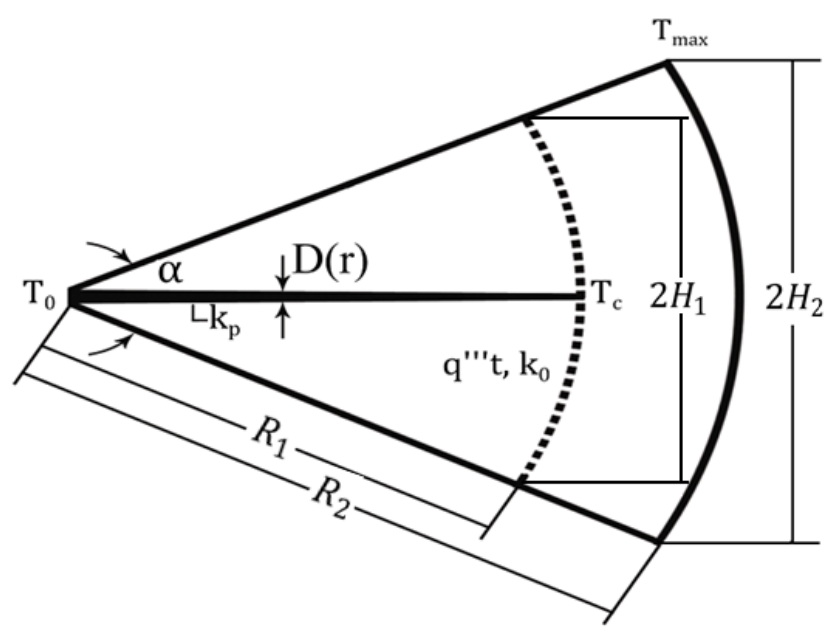

Fig. 2 A sector of the disc with radial variable cross section configuration of highly conductive material. 
It is assumed that there are many radial inserts so that the sectors are slender enough to be approximated by an isosceles triangles of base $2 H$ and height $R$.

At first, based on the constructal theory, one of these sectors is considered as the elemental volume. Then, its thermal resistance is determined and optimized due to various aspect ratios. The corresponding thermal resistance of the entire disc can be calculated by assembling the sectors together.

As the inserts are considered incomplete, there is a region in the disc where there is no highly conductive insert in it. Thus, the domain can be divided into two regions: the outer region without highly conductive inserts and the central part with the cooling inserts.

\subsubsection{Region without highly conductive material}

Firstly, bear in mind that there is no difference in formulating thermal resistance between constant and variable cross-section highly conductive insert for the outer region where there is no cooling insert. Two-dimensional conduction equation in cylindrical format can be written as,

$\frac{\partial^{2} T}{\partial r^{2}}+\frac{1}{r} \frac{\partial T}{\partial r}+\frac{1}{r^{2}} \frac{\partial^{2} T}{\partial \theta^{2}}+\frac{q^{\prime \prime \prime}}{k_{0}}=0$

In order to solve the above equation, boundary conditions should be specified. As the disc is adiabatic, there is no heat conduction at the edge and $\left(\frac{\partial T}{\partial r}\right)_{\left(r=R_{2}, \theta\right)}=0$, also $\left(\frac{1}{r} \frac{\partial T}{\partial \theta}\right)_{(r, \theta=0)}=0$ and $\left(\frac{1}{r} \frac{\partial T}{\partial \theta}\right)_{(r, \theta=\alpha)}=0$ due to symmetry of the problem where $\theta_{0}$ is the sectional degree. Since highly conductive material is responsible for the main part of the heat flux conduction, it is approximated that the direction of the heat flux at $r \leq R_{1}$ is perpendicular to the insert. Thus, $T\left(r=R_{1}, \theta\right) \approx f(\theta)$ is the fourth boundary condition. It should be noted that this assumption is also implemented in (Rocha et al., 2002) to obtain the temperature difference between the heat sink and the highly conductive inserts connected to the rim of the disc. Moreover, in section 4.3, consistency of this assumption is checked by solving the problem numerically in a 2D format. Comparing the results of numerical and analytical solutions, similar behavior and acceptable agreement between them are observed.

With the above boundary conditions, using change of variables to homogenize the equation, then separating variables and finally nondimensionalizing the maximum temperature difference, it can be concluded that,

$\tilde{T}_{1}=\frac{\mathrm{T}_{\max }-\mathrm{T}_{0}}{\mathrm{q}^{\prime \prime \prime} \mathrm{A}_{1} / \mathrm{k}_{0}}=\frac{1}{3} \frac{H_{1}}{R_{1}}-$

$\sum_{n=1}^{\infty} \frac{4(-1)^{n}}{(n \pi)^{2}}\left[\frac{\left(R_{2} / R_{1}\right)^{\lambda_{n}}}{1+\left(R_{2} / R_{1}\right)^{2 \lambda_{n}}}\right] \frac{H_{1}}{R_{1}}-\frac{1}{4}\left[\left(\frac{R_{2}}{R_{1}}\right)^{2}-1\right] \frac{R_{1}}{H_{1}}$

$+\frac{1}{2}\left(\frac{R_{2}}{R_{1}}\right)^{2} \ln \left(\frac{R_{2}}{R_{1}}\right) \frac{R_{1}}{H_{1}}$

where $A_{1}=H_{1} R_{1}$ and $\lambda_{n}=n \pi / \alpha$ are the characteristic values.

\subsubsection{Region with highly conductive material}

In order to analyze conduction along the $k_{p}$ blade in this region, first notice that the heat current which flows toward the center increases from $q=0$ at $r=R_{2}$ to the total current of $q=q^{\prime \prime \prime} t A$ at $r=0$; where $q^{\prime \prime \prime}$ is the volumetric heat generation, $A$ is the total area of the disc and $t$ is the thickness of the disc. The increase experienced by $q$ at an intermediate position, $r$, is,

$-d(q)=h q^{\prime \prime \prime} t d r$

Where $h q^{\prime \prime \prime} t$ is the amount of heat current gathered over the vertical surface $h t$ and $h=\left(H_{1} / R_{1}\right) r$ considering an isosceles triangle to represent the sector. The relation between heat and local temperature gradient is,

$q=k_{p} \frac{D}{2} t \frac{d T}{d r}$

Eliminating $q$ between Eqs. (3) and (4) we encounter a second order differential equation with following boundary conditions,

$T=T_{0}$ at $r=0$

$\frac{q^{\prime \prime \prime} \pi\left(R_{2}^{2}-R_{1}^{2}\right) t}{2 N}=k_{p} \frac{D}{2} t \frac{d T}{d r}$ at $r=R_{1}$

Now two different cases can be pursued: constant and variable cross-section inserts. For the constant cross-section, $\mathrm{D}=$ cte, the resultant second order differential equation can be integrated twice and by invoking boundary conditions, Eqs. (5) and (6), specifying $T=T_{R_{1}}$ at $r=R_{1}$ and nondimensionalizing the temperature difference between $T_{R_{1}}$ and $T_{0}$, it can be concluded that,

$\tilde{T}_{2(c t e)}=\frac{T_{R_{1}}-T_{0}}{q^{\prime \prime \prime} A_{1} / k_{0}}=\frac{1}{\tilde{k} \phi}\left(\frac{R_{1}}{R_{2}}\right)^{2}\left(\frac{R_{1}}{H_{1}}\right)\left[\left(\frac{R_{2}}{R_{1}}\right)^{2}-\frac{1}{3}\right]$

For the variable cross-section highly conductive insert, $D=D(r)$, by integrating twice from the differential equation:

$T_{R 1}-T_{0}=\int_{0}^{R_{1}} \frac{A}{D(r)} d r$

where

$A=\frac{-2}{k_{p}}\left[q^{\prime \prime \prime} \frac{r^{2}}{2} \frac{H_{1}}{R_{1}}+c_{1}\right]$ where $c_{1}$ :

$c_{1}=-\left[\frac{q^{\prime \prime \prime} \pi\left(R_{2}^{2}-R_{1}^{2}\right)}{2 N}+\frac{q^{\prime \prime \prime}\left(H_{1} R_{1}\right)}{2}\right]$

Also the volume fraction of highly conductive material to the whole sector is,

$\phi=\frac{1}{H_{2} R_{2}} \int_{0}^{R_{1}} D(r) d r$

It is noted that $\phi$ is the constraint of the problem. Using variational calculus, function of $D(r)$ can be determined with respect to this constraint, Eq. (10), such that Eq. (8) is optimized,

$D(r)=c\left[\left(R_{1}\right)^{2}-(r)^{2}\right]^{\frac{1}{2}}$

Substituting Eq.(11) into constraint (10), the constant factor $c$ can be computed and we will have,

$$
\begin{aligned}
& D(r)=\frac{2 \phi_{1} H_{1}\left(R_{1} / R_{2}\right)^{2}}{\left[\left(R_{1} / R_{2}\right) \sqrt{1-\left(R_{1} / R_{2}\right)^{2}}+\sin ^{-1}\left(R_{1} / R_{2}\right)\right]} \\
& \times\left[\left(R_{2} / R_{1}\right)^{2}-\left(r / R_{1}\right)^{2}\right]^{\frac{1}{2}}
\end{aligned}
$$

Which $\phi=\frac{N D R_{1}}{\pi R_{2}^{2}}=\phi_{1}\left(\frac{R_{1}}{R_{2}}\right)^{2}$ and when $R_{1} / R_{2}$ approaches to unity, i.e. a complete insert, the result will become equal the one reported in [14] as,

$(D(r))_{\text {complete insert }}=\frac{4}{\pi} \phi H\left[1-(r / R)^{2}\right]^{\frac{1}{2}}$

Similar to the procedure implemented for constant- $D$ assumption, The thermal resistance is calculated by substituting Eq. (12) into Eq. (8) and nondimensionalizing it,

$\tilde{T}_{2(\text { variable })}=\frac{\mathrm{T}_{R_{1}}-\mathrm{T}_{0}}{\mathrm{q}^{\prime \prime \prime} \mathrm{A}_{1} / \mathrm{k}_{0}}=\frac{1}{4 \tilde{k} \phi_{1}}\left(\frac{R_{2}}{R_{1}}\right)^{4}\left(\frac{R_{1}}{H_{1}}\right)$

$\times\left[\left(\frac{R_{1}}{R_{2}}\right) \sqrt{1-\left(\frac{R_{1}}{R_{2}}\right)^{2}}+\sin ^{-1}\left(\frac{R_{1}}{R_{2}}\right)\right]^{2}$

In section 4.1, it is shown that the total thermal resistance which is determined by Eq. (2) plus Eq. (7) for constant cross-section and Eq. (2) plus (14) for variable one, has an optimum point due to 
$\left(H_{1} / R_{1}\right)$ provided other parameters are kept constant. Thus, $\left(H_{1} / R_{1}\right)_{\text {opt }}$ is the value at which optimum thermal resistance occurs.

Finally, The corresponding thermal resistance of the entire disc is obtained by using $A_{d i s c}$ instead of $A_{1}$ in Eqs.(2) and (7),

$\tilde{T}_{G R, o p t}=\frac{T_{\max }-T_{0}}{q^{\prime \prime \prime} \pi R_{2}^{2} / k_{0}}=\left(\frac{\tilde{T}}{\pi}\right)\left(\frac{R_{1}}{R_{2}}\right)^{2}\left(\frac{H_{1}}{R_{1}}\right)_{o p t}$

\subsection{Fractal pattern}

Now, we consider the elemental construct of a fractal network of highly conductive structure as shown in Fig. 3. To design tree-shaped incomplete inserts, the results derived for radial configuration will be benefited from. It is noticeable that as the boundary conditions for the stem are different, the result of section 3.1 cannot be used directly for the total fractal configuration. Thus, the domain should be divided into two major parts: the most outer region where there is no highly conductive insert plus the region where tributaries are embedded in and the central part where the insert's configuration is radial. Fig. 3 shows these three regions with three concentric circle slices.

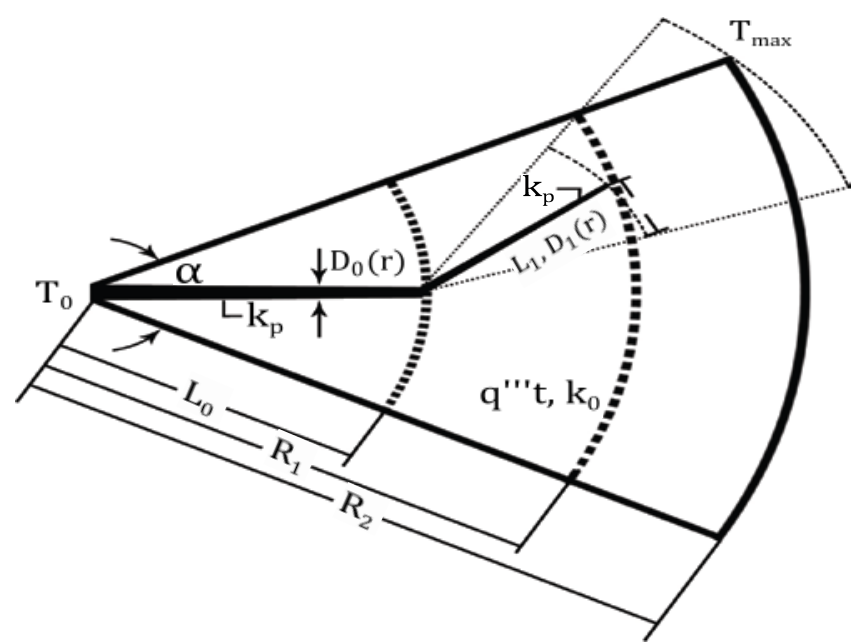

Fig. 3 A sector of the disc with branching variable cross section configuration of highly conductive material.

\subsubsection{Outer region}

As was mentioned previously, derived formula for calculating thermal resistance for radial configuration can be used for this region. Only keep in mind that some notations need to be changed as the region boundaries are different in this case. Thus, to express the thermal resistance of outer region using the radial configuration result, beneath changes are to be done in the notations,

$R_{1}$ (section 3.1) $\rightarrow L_{1}$ (here), $D / 2 \rightarrow D_{1} / 2, T_{0} \rightarrow T_{c}$,

$R_{2} \rightarrow\left(R_{2}-R_{1}+L_{1}\right)$

Thus, $\left(H_{1} / R_{1}\right)$ is replaced by $\left(H_{1} / L_{1}\right)$, and the following relations for the portion between $L_{0}$ and $R_{2}$ can be used,

$\left(\frac{H_{1}}{L_{1}}\right)=\left(\frac{H_{1}}{L_{1}}\right)_{\text {opt }}$ (section. 3.1),

$\phi_{1}=\frac{D_{1}}{H_{1}}, A_{1}=H_{1} L_{1}$

As the result, $D_{1}(r)$ would be,

$D_{1}(r)=\frac{2 \phi_{1} H_{1}\left(L_{1} / M\right)^{2}}{\left[\left(L_{1} / M\right) \sqrt{1-\left(L_{1} / M\right)^{2}}+\sin ^{-1}\left(L_{1} / M\right)\right]}$

$\left[\left(M / L_{1}\right)^{2}-\left(r / L_{1}\right)^{2}\right]^{\frac{1}{2}}$

$$
\begin{aligned}
\left(\frac{M}{L_{1}}\right) & =1+\left(\frac{R_{2}}{L_{1}}\right)-\left(\frac{R_{1}}{L_{1}}\right) \\
& =1+\tilde{R}_{1}\left(\frac{H_{1}}{L_{1}}\right)_{o p t}^{\frac{1}{2}}\left[\left(\frac{R_{2}}{R_{1}}\right)-1\right]
\end{aligned}
$$

Applying above notations in the equation derived previously in section 3.1, the thermal resistances corresponding to both regions, the one without highly conductive inserts plus region with tributary part, for constant and variable cross-section inserts are expressed by Eqs. (20) and (21), respectively.

$$
\begin{aligned}
& \tilde{T}_{1(\text { cte })}=\frac{T_{\text {max }}-T_{c}}{q^{\prime \prime \prime} A_{1} / k_{0}}= \\
& \frac{1}{3} \frac{H_{1}}{L_{1}}-\sum_{n=1}^{\infty} \frac{4(-1)^{n}}{(n \pi)^{2}}\left[\frac{\left(M / L_{1}\right)^{\lambda_{n}}}{\left.1+\left(M / L_{1}\right)^{2 \lambda_{n}}\right]} \frac{H_{1}}{L_{1}}\right. \\
& -\frac{1}{4}\left[\left(\frac{M}{L_{1}}\right)^{2}-1\right] \frac{L_{1}}{H_{1}}+\frac{1}{2}\left(\frac{M}{L_{1}}\right)^{2} \frac{L_{1}}{H_{1}} \ln \left(\frac{M}{L_{1}}\right) \\
& +\frac{1}{\tilde{k} \phi_{1}}\left(\frac{L_{1}}{H_{1}}\right)\left[\left(\frac{M}{L_{1}}\right)^{2}-\frac{1}{3}\right] \\
& \tilde{T}_{1(\text { variable })}=\frac{T_{\max }-T_{c}}{q^{\prime \prime \prime} A_{1} / k_{0}}= \\
& \frac{1}{3} \frac{H_{1}}{L_{1}}-\sum_{n=1}^{\infty} \frac{4(-1)^{n}}{(n \pi)^{2}}\left[\frac{\left(M / L_{1}\right)^{\lambda_{n}}}{1+\left(M / L_{1}\right)^{\lambda_{n}}}\right] \frac{H_{1}}{L_{1}} \\
& -\frac{1}{4}\left[\left(\frac{M}{L_{1}}\right)^{2}-1\right] \frac{L_{1}}{H_{1}}+\frac{1}{2}\left(\frac{M}{L_{1}}\right)^{2} \frac{L_{1}}{H_{1}} \ln \left(\frac{M}{L_{1}}\right) \\
& +\frac{1}{4 \tilde{k} \phi_{1}}\left(\frac{L_{1}}{H_{1}}\right)\left(\frac{M}{L_{1}}\right)^{4} \\
& \times\left[\left(\sin ^{-1}\left(\frac{L_{1}}{M}\right)\right)+\left(\frac{L_{1}}{M}\right)\left(1-\left(\frac{L_{1}}{M}\right)^{2}\right)^{1 / 2}\right]^{2}
\end{aligned}
$$

\subsubsection{Central part}

As the boundary conditions for this part are different, we cannot use $\left(H_{1} / L_{1}\right)_{o p t}$ for the $\left(H_{0} / L_{0}\right)$ obtained from section 3.1 ; instead, it can be written as,

$\left(\frac{H_{0}}{L_{0}}\right) \cong\left(\frac{\alpha}{2}\right), \quad A_{0}=H_{0} L_{0}$

where $\alpha$ is the tip angle (Fig. 2) which is the function of other geometric parameters, i.e. the number of $L_{1}$ or $A_{1}$ elements, $\mathrm{N}$, and the number of branches $L_{1}$ which is connected to the radial inserts, $n$. thus,

$\alpha=\frac{2 \pi n}{N}=\frac{2 \pi n}{2 \pi R_{1} / 2 H_{1}}=\frac{2 n}{\tilde{R}_{1}}\left(\frac{H_{1}}{L_{1}}\right)_{o p t}^{1 / 2}$

In Eq. (23), $\tilde{R}_{1} \equiv R_{1} / A_{1}^{1 / 2}$ and the area $A_{0}$ of the central sector is,

$A_{0} \cong n \widetilde{R}_{1}\left(\frac{H_{1}}{L_{1}}\right)_{o p t}^{1 / 2} A_{1}\left[1-\frac{1}{\tilde{R}_{1}}\left(\frac{H_{1}}{L_{1}}\right)_{o p t}^{-1 / 2}\right]^{2}$

Again, two scenarios can be considered for the cross-section, i.e. variable and constant. In the case that $D_{0}$ and $D_{1}$ are assumed constant, the temperature difference between $T_{c}$ and $T_{0}$ is determined by eliminating $q$ between Eqs. (3) and (4), then integrating twice from the resultant differential equation, invoking $T=T_{0}$ at $r=0$ and another boundary condition from the radial pattern which is,

$$
\begin{aligned}
k_{p} D_{0}\left(L_{0}\right) t\left(\frac{d T}{d r}\right)_{r=L_{0}} & \\
& \cong q^{\prime \prime \prime} t\left[n A_{1}+\left(\frac{\alpha}{2}\right)\left(R_{2}^{2}-R_{1}^{2}\right)\right]
\end{aligned}
$$


This boundary condition expresses that the heat generated in the outer region is gathered and transformed to the heat sink in the center by means of stem embedded in the central region. The temperature difference for constant $-D$ assumption is then nondimensionalized and as specified before, $T=T_{c}$ at $=R_{1}$; hence,

$\tilde{T}_{2(c t e)}=\frac{T_{c}-T_{0}}{q^{\prime \prime \prime} A_{1} / k_{0}}=\frac{\left[\tilde{R}_{1}-\left(\frac{L_{1}}{H_{1}}\right)_{o p t}^{1 / 2}\right]}{\tilde{k} \phi_{1} \widetilde{D}}\left(\frac{L_{1}}{H_{1}}\right)_{o p t}^{1 / 2}$

$\times\left[\frac{2}{3} n \tilde{R}_{1}\left(\frac{H_{1}}{L_{1}}\right)_{o p t}^{1 / 2}\left[1-\frac{\left(L_{1} / H_{1}\right)_{o p t}^{1 / 2}}{\tilde{R}_{1}}\right]^{2}+n+n \tilde{R}_{1}\left(\frac{H_{1}}{L_{1}}\right)_{o p t}^{1 / 2}\right.$

$\left.\times\left[\left(\frac{R_{2}}{R_{1}}\right)^{2}-1\right]\right]$

For variable cross-section highly conductive insert, after integrating twice from the differential equation and invoking above boundary conditions, we have,

$T(r)-T_{0}=\int_{0}^{r} \frac{-1}{k_{p} D_{0}(r)}\left[\left(\frac{H_{0}}{L_{0}}\right) q^{\prime \prime \prime} r^{2}+c_{1}\right] d r$,

$c_{1}=-q^{\prime \prime \prime}\left[\left(\frac{H_{0}}{L_{0}}\right)\left(R_{2}^{2}-R_{1}^{2}\right)+n A_{1}+A_{0}\right]$

Again, volume fraction is the constraint,

$\phi=\frac{A_{p}}{\pi R_{2}^{2}}$

$=\frac{1}{\pi R_{2}^{2}}\left[N \int_{0}^{L_{1}} D_{1}(r) d r+(N / n) \int_{0}^{L_{0}} D_{0}(r) d r\right]$

Relation for $\phi$ can be improved further as,

$\phi=\left(\frac{L_{1}}{H_{1}}\right)^{1 / 2} \tilde{R}_{1}^{(-1)}\left(\frac{R_{1}}{R_{2}}\right)^{2} \phi_{1}$

$+\left(\frac{L_{1}}{H_{1}}\right)^{1 / 2} \tilde{R}_{1}\left(\frac{1}{n R_{2}^{2}}\right) \int_{0}^{L_{0}} D_{0}(r) d r$

$\phi_{1}=\frac{1}{H_{1} L_{1}} \int_{0}^{L_{1}} D_{1}(r) d r$

Now, optimizing Eq. (27) regarding to Eq. (29), we have,

$D_{0}(r)=$

$$
\left(\frac{2}{C_{1} C_{2}}\right)\left(\frac{R_{2}}{R_{1}}\right)^{2} \frac{\left(\phi-C_{3} \phi_{1}\right) H_{0}}{C_{4}^{2}}\left[C_{4}^{2}-\left(\frac{r}{L_{0}}\right)^{2}\right]^{1 / 2}
$$

where

$C_{1}=\sin ^{-1}\left(1 / C_{4}\right)+\left(1 / C_{4}\right) \sqrt{1-\left(1 / C_{4}\right)^{2}}$,

$C_{2}=\left[1-\frac{1}{\tilde{R}_{1}}\left(\frac{L_{1}}{H_{1}}\right)^{1 / 2}\right]^{2}, C_{3}=\frac{1}{\tilde{R}_{1}}\left(\frac{L_{1}}{H_{1}}\right)^{1 / 2}\left(\frac{R_{1}}{R_{2}}\right)^{2}$,

$C_{4}=\left(1+\frac{1}{\tilde{R}_{1} C_{2}}\left(\frac{L_{1}}{H_{1}}\right)^{\frac{1}{2}}+\frac{1}{C_{2}}\left[\left(\frac{R_{2}}{R_{1}}\right)^{2}-1\right]\right)^{1 / 2}$

Substituting Eq. (30) into Eq. (27) and nondimensionalizing it, the thermal resistance for this region can be expressed as,

$\tilde{T}_{2(\text { variable })}=\frac{T_{c}-T_{0}}{q^{\prime \prime \prime} A_{1} / k_{0}}$

$=\left(\frac{2}{\alpha}\right)\left(\frac{R_{1}}{R_{2}}\right)^{2} \frac{C_{1} C_{2} C_{4}^{2}}{2 \tilde{k}\left(\phi-C_{3} \phi_{1}\right)} \times$

$\left[\left(\tilde{R}_{1}^{2}\left(\frac{\alpha}{2}\right)\left(\frac{R_{2}}{R_{1}}\right)^{2}\left[1-\left(\frac{R_{1}}{R_{2}}\right)^{2}\right]+n\right) \sin ^{-1}\left(\frac{1}{C_{4}}\right)\right.$

$\left.+\left(\frac{A_{0}}{A_{1}}\right)\left(\sin ^{-1}\left(1 / C_{4}\right)-\left(\frac{C_{4}^{2}}{2}\right) C_{1}^{*}\right)\right]$

where $C_{1}^{*}$ is the conjugate of $C_{1}$.
Furthermore, the global thermal resistance for branching pattern, $\tilde{T}_{G B, o p t}$, can be written as,

$\tilde{T}_{G B, o p t}=\frac{T_{\max }-T_{0}}{q^{\prime \prime \prime} \pi R_{2}^{2} / k_{0}}=\left(\frac{\tilde{T}}{\pi \tilde{R}_{1}^{2}}\right)\left(\frac{R_{1}}{R_{2}}\right)^{2}$

\section{RESULT AND DISCUSSION}

Thermal resistance of radial and fractal configurations are analyzed in this section. For each structure, more attention is paid to the width of the highly conductive insert cross-section. Moreover, the thermal resistance of variable cross-section is compared with that of constant cross-section configuration for different conditions. In a nutshell, it is concluded that variable cross-section always decreases the thermal resistance but when the complexity of the control volume increases, the percentage of decrease in thermal resistance diminishes. Worth to mention that even in the latter case, the result is significant in the event that expense of highly conductive material comes to picture.

\subsection{Radial pattern}

The optimum width of cross-section for radial pattern is obtained using Eq. (12) as a function of the length of the insert keeping other parameters as constants, Fig. 4. This figure is sketched for different $R_{1} / R_{2}$ ratios. Since the amount of highly conductive material allocated to the element is assumed to be constant $(\phi=c t e)$, it is obvious that the mean $D_{\text {opt }}(r)$ decreases as $R_{1} / R_{2}$ increases.

Figure 5 shows the thermal resistance of constant $-D$ and $D=D(r)$ configurations for different $R_{1} / R_{2}$ ratios. A similar behavior can be observed in this figure, i.e. in both cases, constant and variable cross-sections, there is an optimum point due to aspect ratio of the elemental construct, $H_{1} / R_{1}$. Also, as $R_{1} / R_{2}$ increases, the decrease in thermal resistance is more obvious.

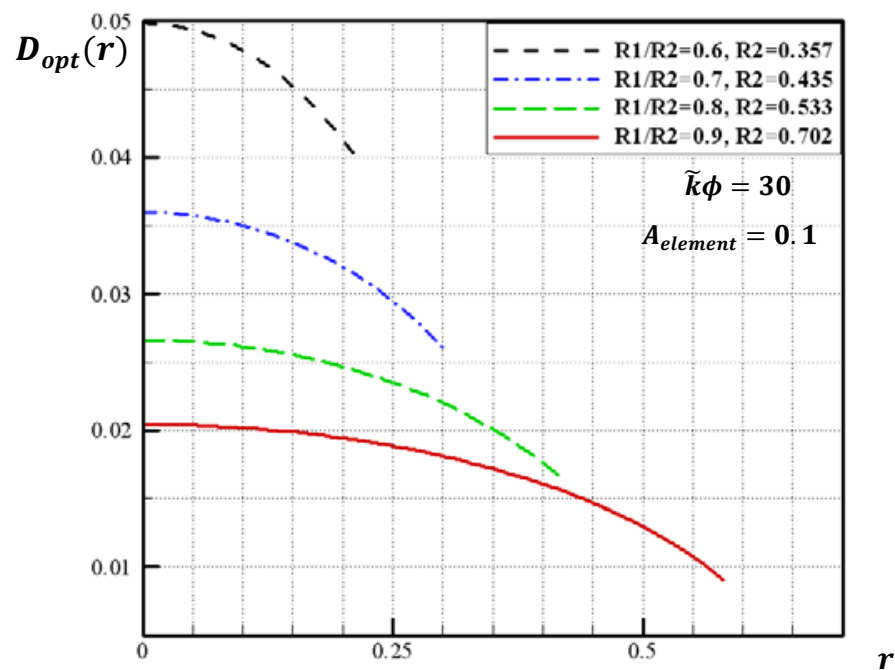

Fig. $4 D_{\text {opt }}(r)$ at different $R_{1} / R_{2}$.

Because of the complexity of Eq. (2) plus (7) and Eq. (2) plus (14), it is hard to obtain an analytic relation for optimum thermal resistance. Thus, optimum aspect ratios and optimum thermal resistances are determined by solving the equations numerically as shown in Fig. $6(a, b)$. These figures illustrate that the decrease in both of the optimum values intenifies as $R_{1} / R_{2}$ increases and $\tilde{k} \phi$ decreases. The reason for this behavior lays in the fact that by increasing the $R_{1} / R_{2}$ ratio, the difference between optima of variable 


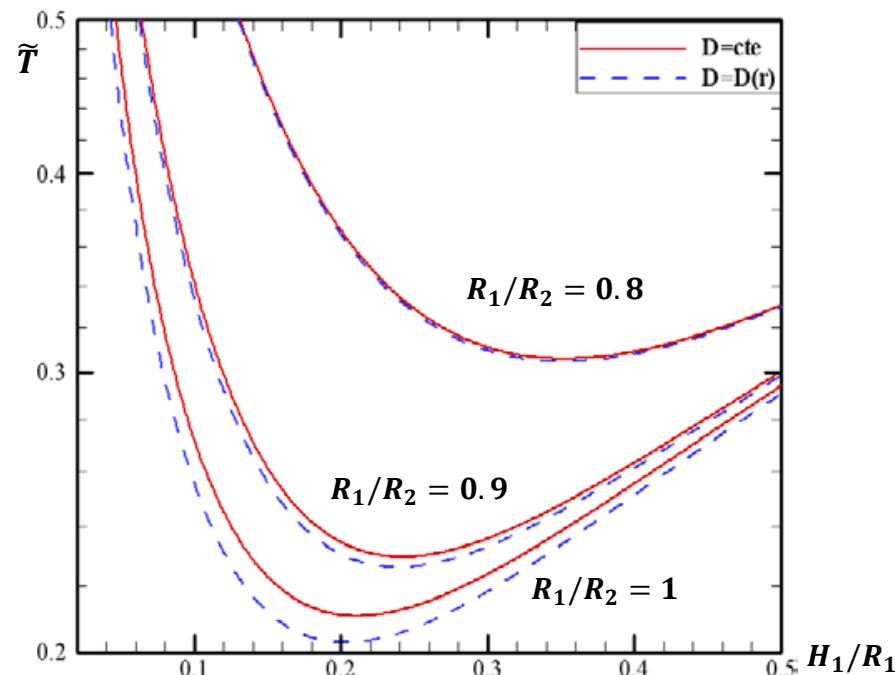

Fig. 5 Thermal resistance for different values of $R_{1} / R_{2}$

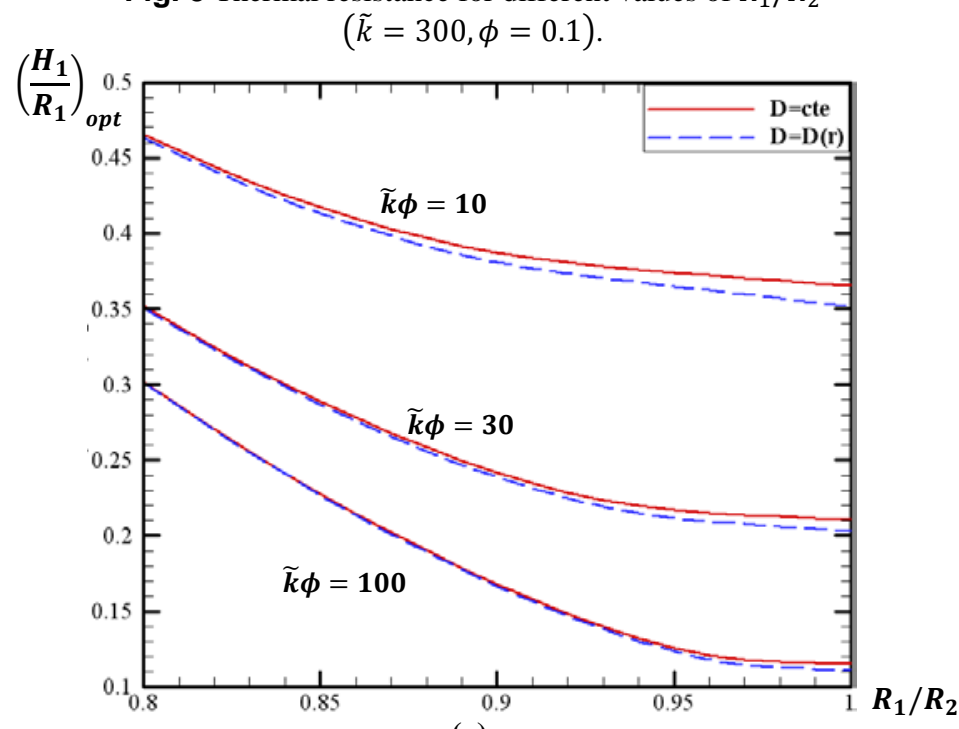

(a)

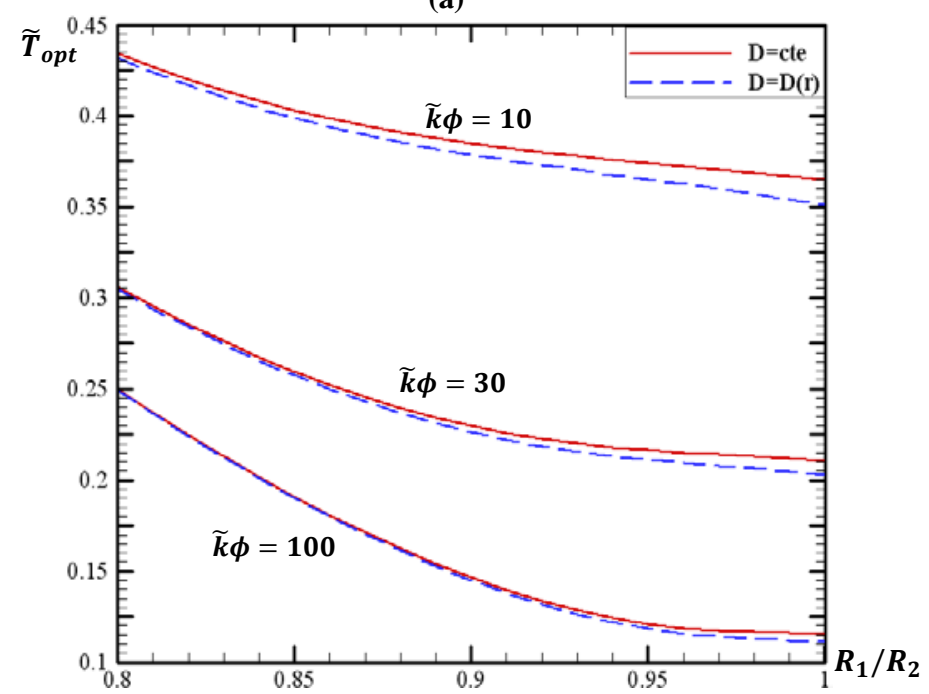

(b)

Fig. 6 (a) Optimum aspect ratio and (b) optimum thermal resistance for different values of $\tilde{k} \phi$. and constant cross-section inserts grows and the increment of $\tilde{k} \phi$ causes the optimum thermal resistance to become less dependent upon the shape of the inserts. Also, it is clear from Fig. 6 that as $\tilde{k} \phi$ and $R_{1} / R_{2}$ increase, the optimum aspect ratios and thermal resistances decrease, expectedly.

\subsubsection{Comparison}

In this section, we try to compare the global thermal resistances of configurations with incomplete and complete inserts while crosssection of highly conductive inserts is considered variable. Also, decrease in global resistance by using of variable cross section highly conductive inserts instead of constant one is investigated. Figure 7 shows the percentage of difference in optimum global thermal resistance between complete and incomplete variable cross-section inserts for different $\tilde{k} \phi$ defined as,

$$
\delta=100 \times\left|\frac{\mathrm{IITR}-\mathrm{CITR}}{\mathrm{CITR}}\right|
$$

where IITR and CITR are incomplete and complete insert thermal resistances, respectively. This figure illustrates that there exists a range of $R_{1} / R_{2}$ where the global thermal resistance of incomplete inserts is less than that of complete one; but this range decreases as $\tilde{k} \phi$ increases. The possible reason is at smaller $\tilde{k} \phi$ s and $R_{1} / R_{2} \approx 1.0$ the cooling power is not efficient enough to change the direction of heat flux considerably to inserts instead of heat sink. Therefore, the optimized condition will be when $R_{1} / R_{2}<1.0$ and as a result, because of the constraint of the problem, the thickness of inserts is proper enough to affect on heat flux, efficiently.

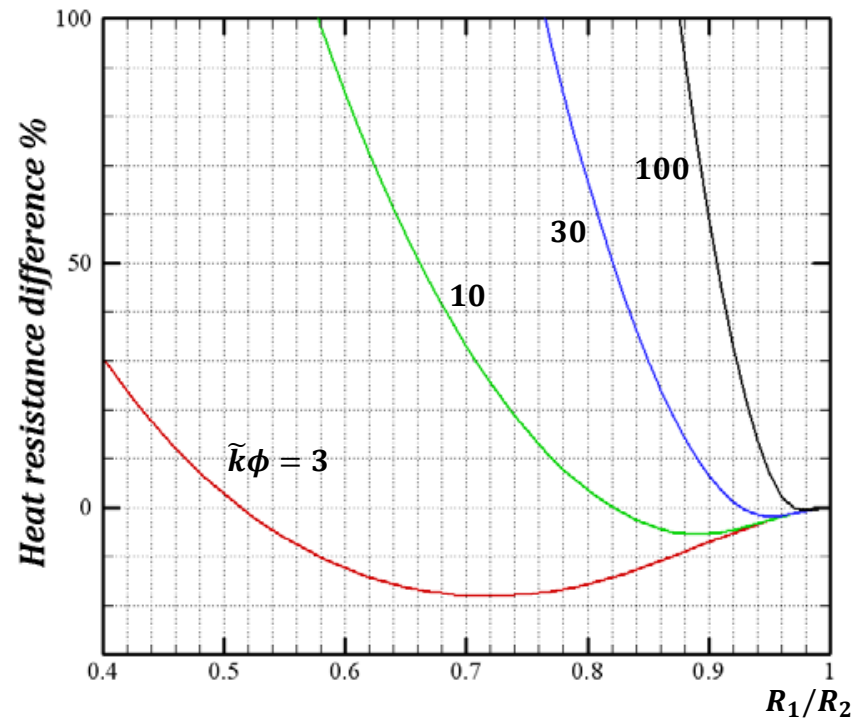

Fig. 7 Difference in optimum global thermal resistance between incomplete and complete inserts $\left(D=D_{\text {opt }}(r)\right)$.

Figure 8 shows the percentage of decrease in optimum global thermal resistance using variable cross-section highly conductive insert defined as,

$\varepsilon=100 \times\left|\frac{V G R-C G R}{\mathrm{CGR}}\right|$

where VGR and CGR are global thermal resistances of variable and constant cross-sections, respectively. This figure illustrates that the percentage of decrease rises as $R_{1} / R_{2}$ increases and $\tilde{k} \phi$ reduces. As a matter of fact, 2 factors, $R_{1} / R_{2}$ and $\tilde{k} \phi$, play significant role here. 


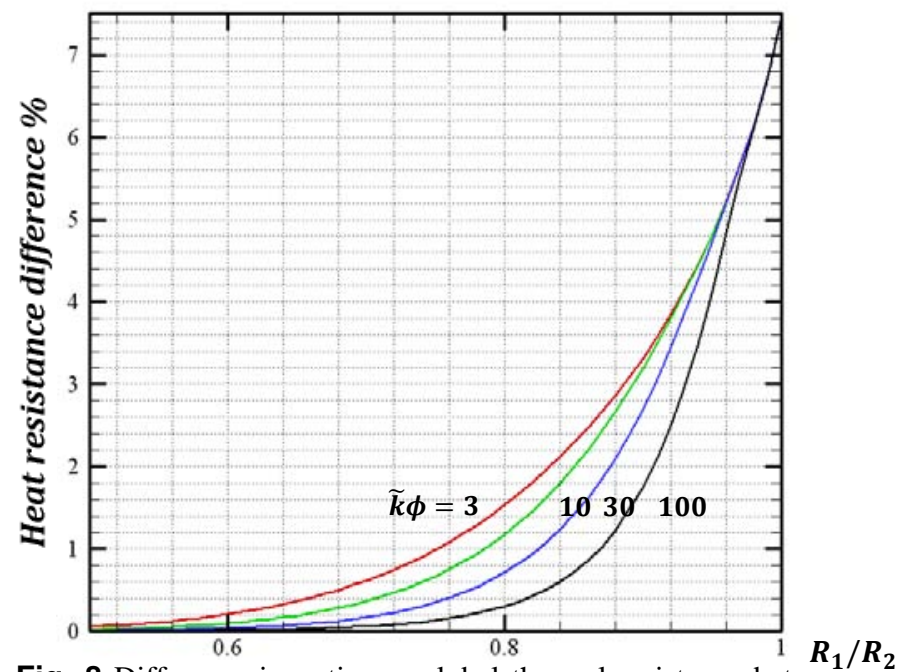

Fig. 8 Difference in optimum global thermal resistance between incomplete inserts; $D=$ cte and $D=D_{\text {opt }}(r)$.

In small $R_{1} / R_{2}$ s, with increasing $\tilde{k} \phi$, the shape of inserts doesn't change the thermal resistance, considerably. In other words, in small $R_{1} / R_{2} \mathrm{~S}$, the factor of $\tilde{k} \phi$ has the dominant impact on performance. Furthermore, at this amount of $R_{1} / R_{2} \mathrm{~s}$, the freedom of maneuver is limited and the shape of inserts doesn't have considerable change, compared with constant $D$.

In larger $R_{1} / R_{2} \mathrm{~S}$, the shape of inserts has the dominant impact due to the fact that the insert length is stretched enough and its shape is completely different from constant conducting path. In this condition, heat resistance difference corresponding to disc with variable and constant inserts is going to be independent of $\tilde{k} \phi$.

The maximum percentage of decrease in global thermal resistance is about $7.4 \%$ which occurs at $R_{1} / R_{2}=1$ independently from $\tilde{k} \phi$.

\subsection{Branching pattern}

The optimum width of highly conductive insert for stem $D_{0}(r)$ and tributary $D_{1}(r)$ which were determined in Eqs. (18) \& (30) are plotted in Fig. 9 for different $\tilde{R}_{1}$. This figure illustrates that $L_{0}$ increases with $\tilde{R}_{1}$, while $L_{1}$ is not so sensible.

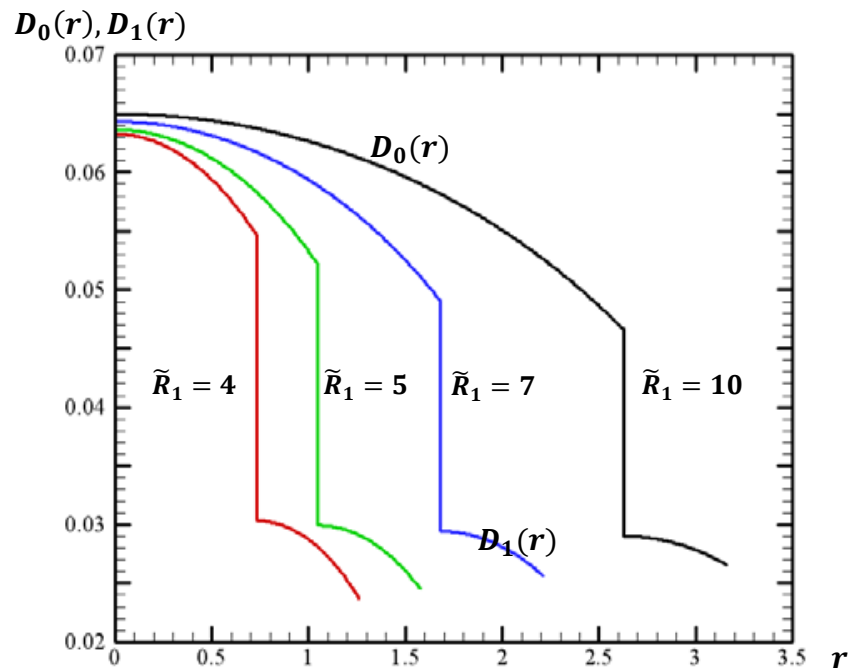

Fig. 9 Optimum width of highly conductive inserts for stem $D_{0}(r)$ and tributary inserts $D_{1}(r)\left(\tilde{k} \phi=30, R_{1} / R_{2}=0.8, A_{1}=0.1\right)$.
Figure 10 shows the thermal resistance for variable and constant cross-section highly conductive inserts using Eqs. (20), (21), (26) \& (32). For all the values of $\phi_{1}$, thermal resistance corresponding to constant $D$ configuration is greater than that of variable cross-section configuration and also they have a behavior similar to the radial pattern.

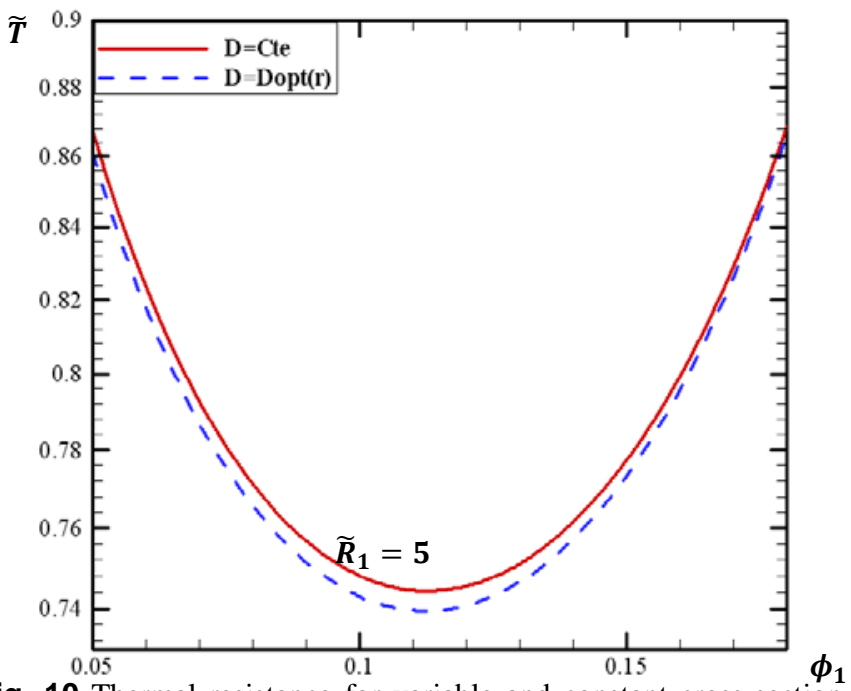

Fig. 10 Thermal resistance for variable and constant cross-section highly conductive inserts at $\tilde{k} \phi=30, R_{1} / R_{2}=0.9$.

It is demonstrated from Fig. 10, the thermal resistance has an optimum due to $\phi_{1}$ but because of the complexity of the equation derived for the thermal resistance at section 3.2, the optimum thermal resistance is determined numerically for both cases, constant and variable cross-sections.

As shown in Fig. 11, for two different $\tilde{k} \phi$ s, variable and constant cross-section configurations have a similar trend and both decrease as $R_{1} / R_{2}$ increases.

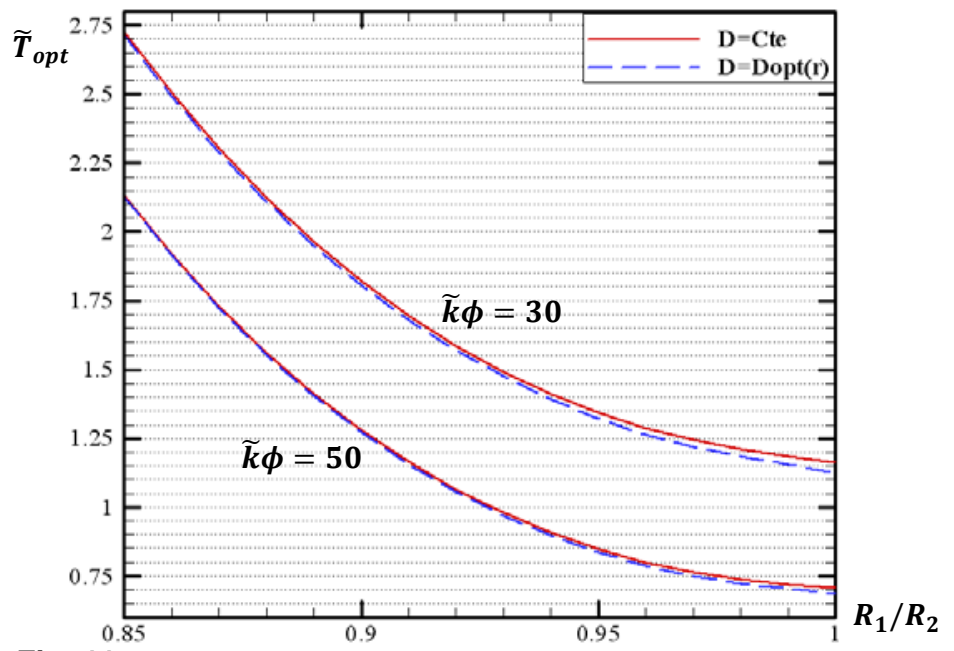

Fig. 11 Optimum thermal resistance for variable and constant crosssection highly conductive inserts at $\tilde{R}_{1}=5$.

Figure 12 shows the percentage of decrease in optimum global thermal resistance when variable cross-section highly conductive inserts are utilized. This figure illustrates that the decrease in this 
value is less in compare with radial patterns and it is concluded that increasing the complexity of the shape when volume is specified, cannot decrease the optimum global resistance anymore. Moreover, it is evident that the decrement of optimum global thermal resistance increases with $\tilde{R}_{1}$. It is physically logical; in fact, with increasing the dimension of disc, the shape of inserts plays more contribution in reducing the thermal resistance, compared to disc with constant thickness of inserts.

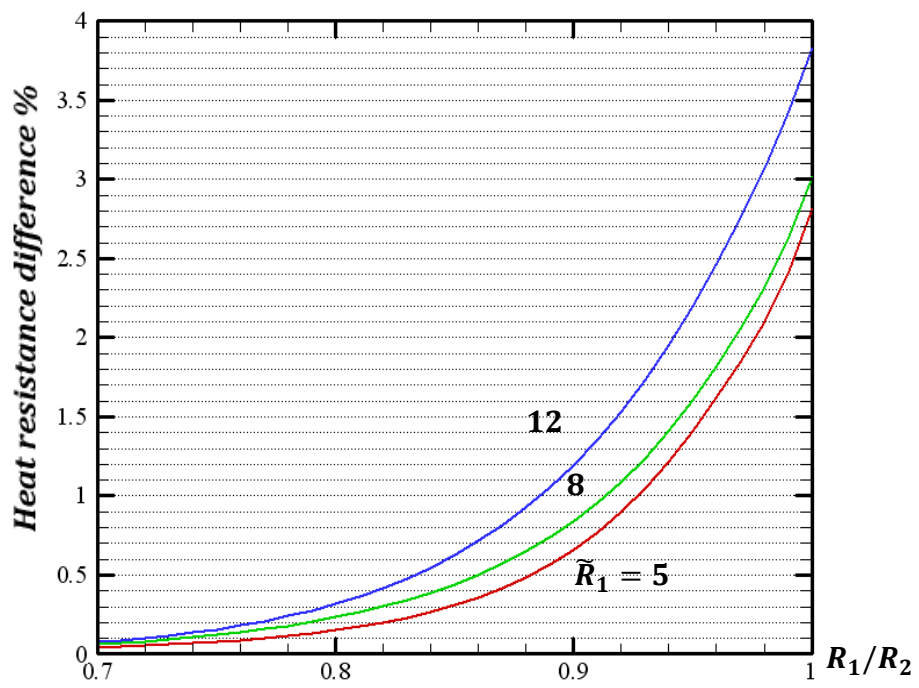

Fig. 12 Decrease in global thermal resistance using variable crosssection highly conductive insert for $\tilde{k} \phi=30$.

\subsection{Numerical results and comparison}

Numerical results presented in this section can be used to validate the analytical solution provided beforehand. Because there is no approximation for the numerical scheme and that the conduction equation is solved in its two dimensional format throughout the whole domain. On the other hand, since the optimum thermal resistances are obtained based on constructal theory, here is a suitable opportunity to examine validity of this strategy. Because, the absolute optimal thermal resistance is obtained here, numerically.

Besides, since the speed of numerical method in solving a certain case is considerably less than analytical one, conscientiously thus it is preferred to use the analytical method if there is an acceptable agreement with the numerical one.

There are two different domains to be solved and an interface between them: the region with high conductivity material and the low conductivity region with volumetric heat generation, $q^{\prime \prime \prime}$. Thus, the steady state conduction equations for these two different regions can be solved, respectively:

$k_{p} \nabla^{2} T=0$

$k_{0} \nabla^{2} T=-q^{\prime \prime \prime}$

Because of the symmetry existed in the geometry, a sector of the disc is solved, numerically. $\partial T / \partial n=0$ is the boundary condition for all the boundaries except the tip of the sector where a condition of constant temperature is specified which in fact is the temperature of the heat sink. Also, $n$ is the normal vector to each boundary. For the interface, it is assumed that the heat flux is conserved while the temperature is equal for both domains at common nodes.

The equations of $2 \mathrm{D}$ conduction are solved numerically using MATLAB partial differential equations toolbox code (MATLAB) with unstructured triangular elements. The appropriate mesh size is obtained by satisfying the convergence criterion of Eq. (38):

$\delta=\left|\frac{\tilde{T}_{\max }^{j}-\tilde{T}_{\max }^{j+1}}{\tilde{T}_{\max }^{j}}\right|<0.001$

where $\widetilde{T}_{\max }^{j+1}$ is the maximum thermal resistance by means of using quadrupled elements relative to $\tilde{T}_{\text {max }}^{j}$. Table. 1 shows the results of the above grid study. It is found that refining three steps is enough to satisfy the convergence criterion and the results would be independent from the mesh size.

Table 1 Grid independency test for $\tilde{R}_{1}=4, N=10.94, \tilde{k}=300$, $\tilde{L}_{0}=1.98, \phi=0.1, R_{1} / R_{2}=0.9$ and $A_{1}=0.05$

\begin{tabular}{|c|c|c|}
\hline Number of elements & $\widetilde{T}_{\max }$ & $\delta$ \\
\hline 3720 & 0.664703 & 0.001882 \\
\hline 14882 & 0.665954 & 0.000544 \\
\hline 59528 & 0.666316 & 0.000178 \\
\hline 238112 & 0.666435 & \\
\hline
\end{tabular}

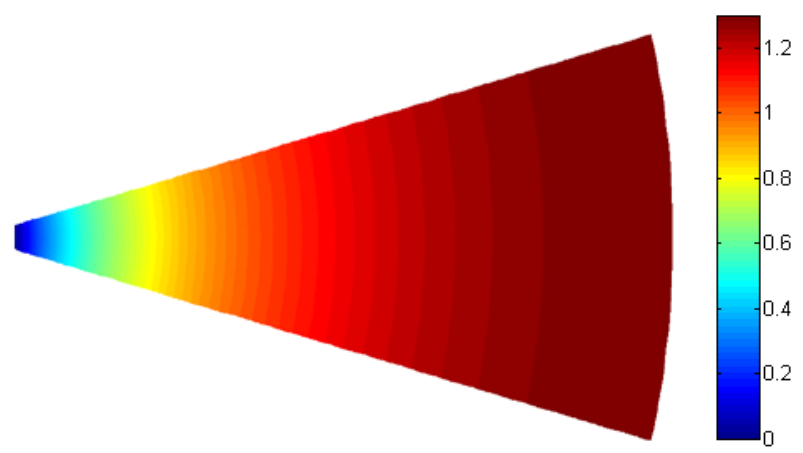

(a)

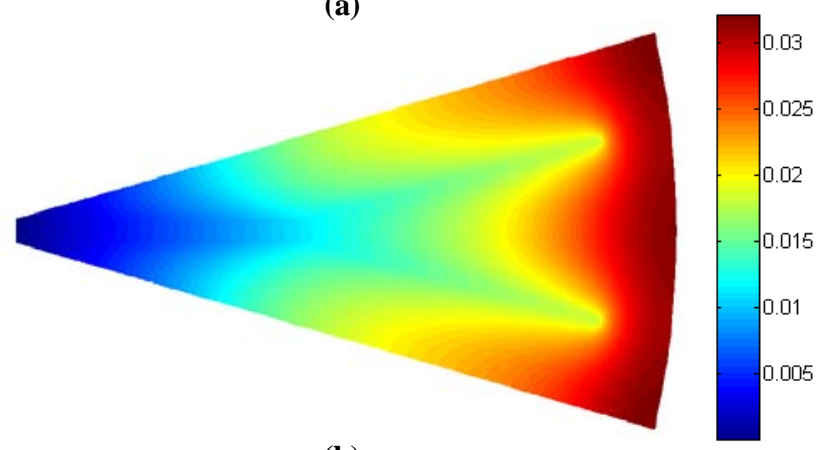

(b)

Fig. 13 Temperature distribution contours for cases (a) without conductive insert $\tilde{T}_{\max }=26.01$ (b) with variable cross-section highly conductive insert $\tilde{T}_{\text {max }}=0.666$ at $\tilde{R}_{1}=4, N=10.94, \tilde{k}=$ 300, $\tilde{L}_{0}=1.9767, \phi=0.1, R_{1} / R_{2}=0.9 \& A_{1}=0.05$.

Figure 13 shows the temperature distribution for a sector (a) without highly conductive material (b) with these inserts incompletely distributed through the sector with two branches. The figure demonstrates that there is a considerable decrease in maximum temperature when highly conductive inserts are used which shows the effectiveness of such a cooling system. To compare these results with analytical solution, all of the parameters are nondimensionalized like section 3.

Figure 14 shows a comparison between numerical results and analytical solution for two $R_{1} / R_{2}$ cases. This figure portrays similar behaviours for two cases. 


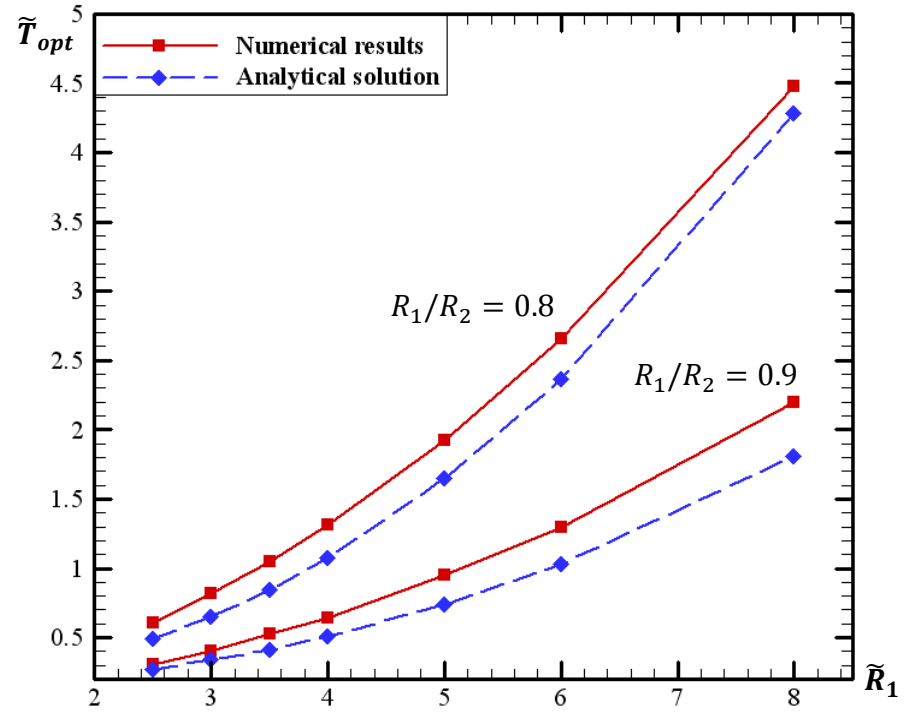

Fig. 14 Numerical results and analytical solution at $\tilde{k}=300$.

Moreover, it is observed from this figure that there is a good consistency between the results specially for the case with $R_{1} / R_{2}=0.8$. The present difference between the numerical and analytical results are completely logical as in the analytical solution we assumed a one-dimensional conducting equation for the region with highly conductive material. These consistent results present the validity of this assumption and also efficiency of constructal theory in optimizing the flow resistances.

\section{CONCLUSION}

Incomplete variable cross-section highly conductive networks for radial and tributary configurations are investigated in this paper. The width of inserts is determined using variational calculus. The thermal resistance for constant and variable cross-section cases is solved analytically and it is shown that there is a decrease in thermal resistance for all conditions when variable cross-section inserts are used. But this increase in the complexity of the problem in tributary case is not as effective as in radial configuration in decreasing the thermal resistance. However, incomplete highly conductive inserts in some specific conditions have an advantage over the complete inserts which gives the same and even lower thermal resistance. Finally, as some approximations were used in analytical solution which the most important of them was the assumption of solving one-dimensional conduction equation for the region with highly conductive material and also the optimization process was carried out based on constructal theory, a numerical solution was performed to validate the analytical results where an acceptable consistency was observed.

\section{NOMENCLATURE}

A area $\left(\mathrm{m}^{2}\right)$

$D \quad$ width of insert (m)

$k_{0} \quad$ thermal conductivity of heat generating material $(\mathrm{W} / \mathrm{m} \mathrm{K})$

$k_{p} \quad$ high-thermal conductivity (W/m K)

$\tilde{k} \quad$ conductivity ratio $\left(k_{p} / k_{0}\right)$

$L \quad$ length of the insert (m)

$n \quad$ number of peripheral elements

$N \quad$ number of sectors

$q \quad$ heat current (W)

$q^{\prime \prime \prime} \quad$ volumetric heat generation rate $\left(\mathrm{W} / \mathrm{m}^{3}\right)$

$R \quad$ radius (m)

$R_{1} \quad$ inner radius (m)

$R_{2} \quad$ outer radius (m)

$\tilde{R} \quad$ dimensionless radius $\left(R_{1} / A_{1}^{1 / 2}\right)$

$t \quad$ thickness of the disc (m)

$T$ temperature (K)

$T_{0} \quad$ sink temperature (K)

$T_{c} \quad$ corner temperature (K)

Greek symbols

$\alpha \quad$ tip angle of the sector

$\phi \quad$ volume fraction of high-conductivity material

$\theta \quad$ angle

$\theta_{0} \quad$ angle of sector

$\lambda_{n} \quad$ characteristic values

\section{Subscripts}

max Maximum

opt Optimum

0 central position

1 position near periphery

\section{REFERENCES}

Almogbel, M., Bejan, A., 2001," Constructal optimization of nonuniformly distributed tree-shaped flow structures for conduction," International Journal of Heat and Mass Transfer 44(22), 4185-4194. http://dx.doi.org/10.1016/S0017-9310(01)00080-1

Bejan, A., 2000," From Heat Transfer Principles to Shape and Structure in Nature: Constructal Theory," Journal of Heat TransferTransactions of the ASME, 122(3), 430-449. http://dx.doi.org/10.1115/1.1288406

Bejan, A., 2008," The constructal law of "designedness" in nature. Meeting the Entropy Challenge," AIP Conference Proceedings, 1033, 207-212.

http://dx.doi.org/10.1063/1.2979031
Bejan, A., 2010," The constructal-law origin of the wheel, size, and skeleton in animal design," American Journal of Physics, 78(7), 692699.

http://dx.doi.org/10.1119/1.3431988

Bejan, A., Badescu, V., Vos, A.D., 2000," Constructal theory of economics structure generation in space and time," Energy Conversion and Management, 41(13), 1429-1451. http://dx.doi.org/10.1016/S0196-8904(00)00038-8

Bejan, A., Lorente, S., 2006," Constructal theory of generation of configuration in nature and engineering," Journal of Applied Physics 100(4).

http://dx.doi.org/10.1063/1.2221896 
Bejan, A., Lorente, S., 2008, Design with constructal theory. John Wiley \& Sons, Hoboken.

Bejan, A., Merkx, G.W., 2007, Constructal Theory of Social Dynamics. Springer, New York.

Dan, N., Bejan, A., 1998," Constructal tree networks for the timedependent discharge of a finite-size volume to one point," Journal of applied physics 84(6), 3042-3050.

http://dx.doi.org/10.1063/1.368458

Ghodoossi, L., Egrican, N., 2004," Conductive cooling of triangular shaped electronics using constructal theory," Energy Conversion and Management 45 (6 ), 811-828. http://dx.doi.org/10.1016/S0196-8904(03)00190-0

Gosselin, L., Bejan, A., 2004," Constructal heat trees at micro and nanoscales," Journal of applied physics, 96(10), 5852-5859. http://dx.doi.org/10.1063/1.1782278

Ledezma, G.A., Bejan, A., Errera, M.R., 1997," Constructal tree networks for heat transfer," Journal of Applied Physics 82(1), 89100.

http://dx.doi.org/+10.1063/1.365853

Mathieu-Potvin, F., Gosselin, L., 2007," Optimal conduction pathways for cooling a heat-generating body: A comparison exercise," International Journal of Heat and Mass Transfer 50(1516), 2996-3006.

http://dx.doi.org/10.1016/j.ijheatmasstransfer.2006.12.020
MATLAB, Partial Differential Equation Toolbox , The MathWorks, Inc., Natick, MA, 2002.,".

Rocha, L.A.O., Lorente, S., Bejan, A., 2002," Constructal design for cooling a disc-shaped area by conduction," International Journal of Heat and Mass Transfer 45(8), 1643-1652. http://dx.doi.org/10.1016/S0017-9310(01)00269-1

Rocha, L.A.O., Lorente, S., Bejan, A., 2006," Conduction tree networks with loops for cooling a heat generating volume," International Journal of Heat and Mass Transfer 49 ((15-16)), 26262635.

http://dx.doi.org/10.1016/j.ijheatmasstransfer.2006.01.017

Wei, S., Chen, L., Sun, F., 2009," The area-point constructal optimization for discrete variable cross-section conducting path," Applied Energy, 86(7-8), 1111-1118. http://dx.doi.org/10.1016/j.apenergy.2008.06.010

Wei, S.H., Chen, L.G., Sun, F.R., 2010," Constructal optimization of discrete and continuous-variable cross-section conducting path based on entransy dissipation rate minimization," Science ChinaTechnological Sciences, 53(6), 1666-1677. 10.1007/s11431-0100121-5

Zhou, S., Chen, L., Sun, F., 2007," Optimization of constructal volume-point conduction with variable cross section conducting path," Energy Conversion and Management 48(1), 106-111. http://dx.doi.org/10.1016/j.enconman.2006.05.007 